\title{
The $\mathrm{CBH}$ characterisation theorem beyond algebraic quantum theory
}

\author{
Chris Heunen $^{\mathrm{a}, 1}$, Aleks Kissinger ${ }^{\mathrm{b}}$ \\ ${ }^{a}$ University of Edinburgh, UK \\ ${ }^{b}$ University of Oxford, UK
}

\begin{abstract}
The $\mathrm{CBH}$ theorem characterises quantum theory within a $\mathrm{C}^{*}$-algebraic framework. Namely, mathematical properties of $\mathrm{C}^{*}$-algebras modelling quantum systems are equivalent to constraints that are information-theoretic in nature: (1) noncommutativity of subalgebras is equivalent to impossibility of signalling; (2) noncommutativity of the whole algebra is equivalent to impossibility of broadcasting; (3) the existence of entangled states is implied by the impossibility of secure bit commitment (with the converse conjectured). However, the $\mathrm{C}^{*}$ algebraic framework has drawn criticism as it already contains much of the mathematical structure of quantum theory such as complex linearity. We address this issue by a generalising $\mathrm{C}^{*}$-algebras categorically. In this framework, equivalence (1) holds, equivalence (2) becomes a strict implication, and implication (3) fails in general. Thus we identify exactly what work is being done by the complex-linear structure of $\mathrm{C}^{*}$-algebras. In doing so, we uncover a richer hierarchy of notions of 'classicality' and 'quantumness' of information than visible in the concrete case.
\end{abstract}

\section{Introduction}

Does information play a significant role in the foundations of physics? This question, often abbreviated 'it from bit' after John Wheeler, has received significant attention, and lies at the root of quantum information theory. The seminal work by Clifton, Bub, and Halvorson [8] isolates quantum theory according to the following three information-theoretic constraints:

- It is impossible to signal information faster than light;

- It is impossible to broadcast an unknown state;

- It is impossible to securely implement bit commitment;

\footnotetext{
Email addresses: chris.heunen@ed.ac.uk (Chris Heunen), aleks.kissinger@cs.ox.ac.uk (Aleks Kissinger)

${ }^{1}$ Supported by EPSRC Fellowship EP/L002388/1.
} 
by linking each of them, respectively, with the following algebraic conditions, characteristic of quantum theory:

- Distinct systems are kinematically independent;

- There exist noncommuting observables;

- There exist entangled, or nonlocal, states.

The first two pairs of properties are proven equivalent; for the third only one implication is proven, and the other conjectured.

However, a criticism often raised against this result is that a $\mathrm{C}^{*}$-algebraic framework, including complex numbers and linearity, is assumed from the start 34 , 4, 2]. In the words of one of the authors himself [30, page 204]:

The characterization theorem we proved assumes a $\mathrm{C}^{*}$-algebraic framework for physical theories, which I would now regard as not sufficiently general in the relevant sense, even though it includes a broad class of classical and quantum theories, including field theories, and hybrid theories with superselection rules.

Recent axiomatisations [20, 27, 28, 24, 6] have to a great extent avoided this problem by starting with a framework which retains only the convex structure of probabilistic states. Nevertheless, these frameworks maintain some remnant of linearity in the form of convexity, which does a great deal of work.

In this paper, we investigate the issue by generalising quantum theory in a different direction, which instead retains only the algebraic structure of interaction between classical and quantum systems, via the $C P^{*}$-construction [12], which enables us to consider abstract $\mathrm{C}^{*}$-algebras in a wide variety of categories beyond the usual examples based on vector spaces. By applying this construction to the category of Hilbert spaces, we obtain algebraic quantum information theory in the usual sense, where objects are (finite-dimensional) $\mathrm{C}^{*}$-algebras and morphisms are completely positive linear maps. However, if we apply this to other categories, we obtain nonstandard models of quantum theory. For example, applying the construction to the category of relations gives a possibilistic model of quantum theory, where probabilities of measurement outcomes are replaced by boolean-valued possibilities ('possible' vs. 'impossible'), in a similar spirit to possibilistic physics as described by Fritz [18] or Schumacher and Westmoreland's modal quantum theory 31].

We phrase the above implications in categorical terms, and show which of them survive and which ones fail. That is, our main contribution is to prove the following (non)implications in this generalised setting:

$$
\begin{aligned}
\text { information theory } & \text { quantum theory } \\
\text { no signalling } & \Leftrightarrow \text { kinematic independence } \\
\text { no broadcasting } & \nLeftarrow \text { noncommutativity } \\
\text { no bit commitment } & \nLeftarrow \text { entanglement }
\end{aligned}
$$


The first equivalence of no-signalling with kinematic independence indeed lifts almost unmodified to any category arising from the $\mathrm{CP}^{*}$-construction. However, in the second case, the commutativity of the algebra of observables merely implies the existence of a broadcasting map (and not vice-versa). Interestingly, this yields finer-grained notions of classicality for the systems in a theory. In particular, we can combine this with the result of [11] to show that, in the case of possibilistic quantum theory, this yields a beautiful group-theoretic hierarchy of classical systems. Rather than requiring the existence of a broadcasting map, we can force a system to be classical by requiring that the algebra associated with the system is commutative or that the partially ordered set of propositions (i.e. the $\mathrm{CP}^{*}$-generalisation of the lattice of projections) is distributive, or both. In the case of quantum theory, either of these is equivalent to the existence of a broadcasting map. However, in possibilistic quantum theory, the following inclusions are strict:

\section{distributive $\mathscr{E}$ commutative $\subsetneq$ commutative $\subsetneq$ broadcasting}

The systems in possibilistic quantum theory are groupoids 21], which are a generalisation of groups where the multiplication is allowed to be partiallydefined. We show that broadcasting, the weakest 'notion of classicality', implies that these groupoids are in fact disjoint unions of groups. Then, stricter notions of classicality yield smaller classes of groups. The 'classicality' hierarchy above corresponds exactly to groupoids arising as disjoint unions of:

$$
\text { locally cyclic groups } \subsetneq \text { abelian groups } \subsetneq \text { groups }
$$

For the case of bit commitment, we first assume that our system is 'quantum enough' to admit secure bit commitment. Namely, we assume that it is described by a noncommutative algebra, which by the second implication is strictly weaker than assuming it is no-broadcasting. We then exhibit a noncommutative system in $\mathrm{CP}^{*}[\mathbf{R e l}]$ which simultaneously admits entanglement and a secure bit commitment protocol. The converse remains an open question.

A notable feature of this work is the convergence of two previously disconnected threads in the foundational study of quantum information. On the one hand, we use many abstract, diagrammatic techniques that originated within the program of categorical quantum mechanics [1, 23, 14] (CQM). Yet in contrast to prior CQM work, which relies heavily on the tensor product to give a notion of 'subsystem, we develop abstract, categorical analogues to subsystems which are more general than tensor factors, and develop concepts such as signalling and broadcasting between such subsystems in the style of algebraic quantum information [25] (AQI). We hope this methodology, which both enables AQIstyle generalised systems and CQM-style diagrammatic manipulations, will find many new applications in areas such as algebraic quantum field theory.

Remark 1.1. Note that we refer to the condition called 'nonlocality' in [8] simply as 'entanglement'. This is to avoid a terminology clash with the related (and now more common) use of the term nonlocality, namely the absence of a locally realistic model for observed correlations. 
The rest of this article is laid out as follows. Section 2 sets up our general categorical framework. Sections 3 then investigate one equivalence each: Section 3 signalling, Section 4 broadcasting, and Section 5 bit commitment. We thank Katriel Cohn-Gordon and Mariami Gachechiladze for their MSc thesis work, supervised by Chris Heunen, on parts of this topic [17, 19].

\section{The $\mathrm{CP}^{*}$-construction}

In this section, we will briefly introduce compact dagger categories and the $\mathrm{CP}^{*}$-construction. We assume familiarity with basic categorical concepts, notably the notion of a symmetric monoidal category (see e.g. [26]).

The $C P^{*}$-construction transforms one symmetric monoidal category into another in a way that mirrors the passage from finite-dimensional Hilbert spaces and linear maps to finite-dimensional $\mathrm{C}^{*}$-algebras and completely positive linear maps. It operates on a certain type of symmetric monoidal category called a compact dagger category, whose definition we now recall.

Definition 2.1. A compact closed category is a symmetric monoidal category $\mathbf{C}$ such that every object $A$ in $\mathbf{C}$ has a dual object $A^{*}$ and morphisms

$$
\varepsilon: A \otimes A^{*} \rightarrow I \quad \eta: I \rightarrow A^{*} \otimes A
$$

satisfying

$$
(\varepsilon \otimes 1) \circ(1 \otimes \eta)=1_{A} \quad 1_{A^{*}}=(1 \otimes \varepsilon) \circ(\eta \otimes 1) .
$$

We will represent morphisms in such a category using string diagrams. For more information we refer to the survey [33]. Objects are depicted as labelled wires with upward directed arrows, and their duals as downward wires:

$$
A \uparrow:=\left|A \quad A^{\downarrow}:=\right| A^{*}
$$

The morphisms $\varepsilon$ and $\eta$ are called caps and cups, drawn as:
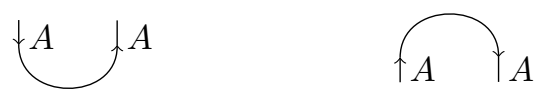

Using this notation, the equations from (11) become:
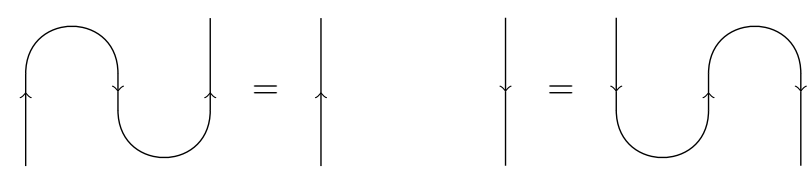

These 'snake equations' embody the relationship between a maximally entangled state (e.g. the Bell state) and the associated effect arising from a Bell measurement. As such, they represent the key to quantum teleportation [1]. 
We can furthermore define the (partial) trace of a morphism $f: X \otimes A \rightarrow$ $X \otimes B$ in a compact closed category via cups and caps:

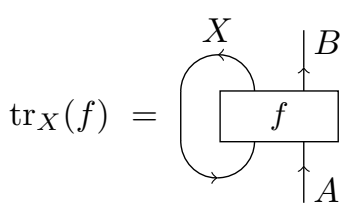

where:

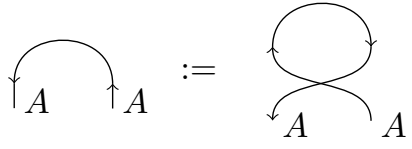

(and similarly for cups).

An important property of compact closed categories is that two morphisms are equal whenever their string diagrams can be deformed into one another. In other words, the only relevant data in a string diagram is its connectivity [33].

Definition 2.2. A dagger category is a category equipped with a contravariant functor $(-)^{\dagger}: \mathbf{C}^{\text {op }} \rightarrow \mathbf{C}$ that satisfies $A^{\dagger}=A$ on objects and $f^{\dagger \dagger}=f$ on morphisms.

Dagger categories enable us to formulate abstract versions of many familiar linear-algebraic concepts. Notably, a morphism in a dagger category is called self-adjoint if $f^{\dagger}=f$ and positive if there exists $g$ such that $f=g^{\dagger} \circ g$. An isomorphism in a dagger category is unitary if $f^{-1}=f^{\dagger}$.

Definition 2.3. A compact dagger category is a category that is both a compact closed category and a dagger category, such that the coherence isomorphisms (associators, unitors, and swap maps) are unitary, and $\varepsilon_{A}^{\dagger}=\eta_{A^{*}}$.

In a compact dagger category, a morphism can take four forms, the morphism itself and its adjoint:

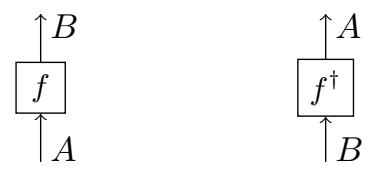

as well as its transpose and conjugate:

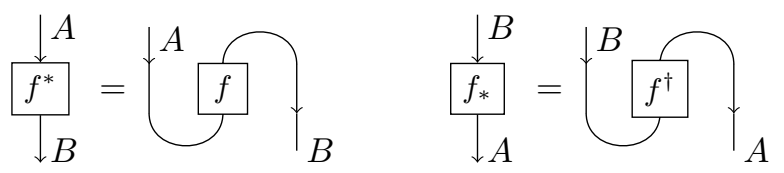


Example 2.4. The category FHilb of finite-dimensional Hilbert spaces and linear maps forms a compact dagger category. The dagger is given by the linear algebraic adjoint:

$$
\left\langle f^{\dagger}(u) \mid v\right\rangle=\langle u \mid f(v)\rangle
$$

The dual $H^{*}$ is the linear algebraic dual, and cups and caps are given respectively as:

$$
\varepsilon(|\psi\rangle \otimes\langle\phi|)=\langle\phi \mid \psi\rangle \quad \eta=\sum\left\langle\phi_{i}|\otimes| \phi_{i}\right\rangle
$$

where $\eta$ does not depend on the choice of orthonormal basis $\left\{\left|\phi_{i}\right\rangle\right\}_{i}$. From this it follows that $f^{*}: K^{*} \rightarrow H^{*}$ is given by pre-composition:

$$
f^{*}(\langle\xi|)=\langle\xi| \circ f
$$

which is sometimes called 'operator transpose'. If we fix a bases for $K$ and $H$, we can identify them with their duals, in which case $f^{*}$ corresponds to matrix transposition.

Example 2.5. The category Rel of sets and relations also forms a compact dagger category. The composition of $R \subseteq A \times B$ and $S \subseteq B \times C$ is the usual composition of relations:

$$
(a, c) \in S \circ R \Longleftrightarrow \exists b \in B:(a, b) \in R,(b, c) \in S
$$

and the monoidal product is given by cartesian product:

$$
\begin{gathered}
A \otimes B=A \times B \\
((a, b),(c, d)) \in R \otimes S \Longleftrightarrow(a, c) \in R,(b, d) \in S
\end{gathered}
$$

Hence the monoidal unit $I=\{*\}$ is a single-element set. The dagger is given by relational converse:

$$
(b, a) \in R^{\dagger} \Longleftrightarrow(a, b) \in R
$$

and cups and caps are:

$$
\eta=\{(*,(a, a)) \mid a \in A\} \quad \varepsilon=\{((a, a), *) \mid a \in A\}
$$

The $\mathrm{CP}^{*}$-construction lets us build a new category whose objects are abstract $\mathrm{C}^{*}$-algebras coming from the original category and whose morphisms are abstract completely positive maps. To do this, we first make precise what we mean by 'abstract $C^{*}$-algebra'.

Definition 2.6. A monoid in a compact dagger category is an object $A$ together with a morphism $\overbrace{\widehat{\gamma}}: A \otimes A \rightarrow A$ and $\hat{\mathrm{O}}: I \rightarrow A$ satisfying
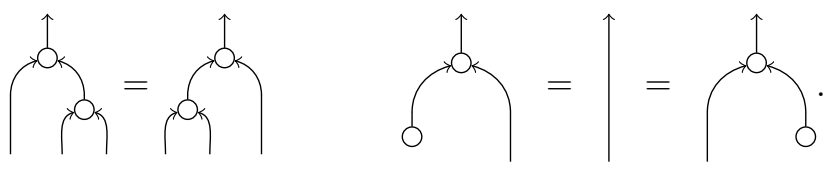
A dagger Frobenius structure is a monoid satisfying

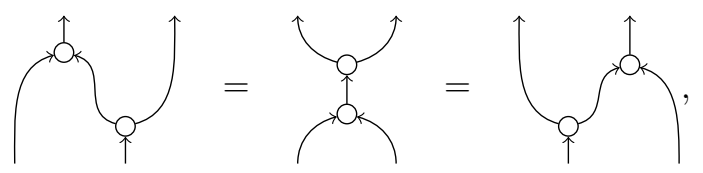

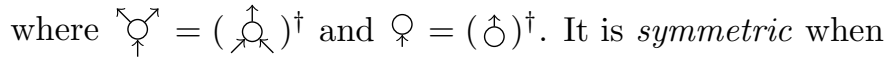

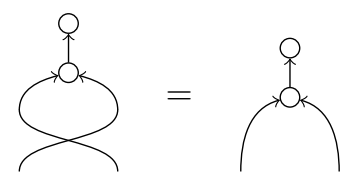

and special when

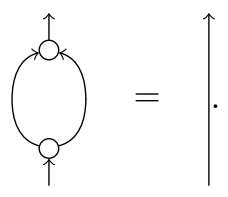

We call a dagger Frobenius structure that is symmetric and special an abstract $C^{*}$-algebra.

It was shown in [35] that dagger Frobenius structures in FHilb correspond exactly to finite-dimensional $\mathrm{C}^{*}$-algebras, and in [22], it was furthermore shown that any such $\mathrm{C}^{*}$-algebra is isomorphic to one that is special and symmetric.

Example 2.7. For a $D$-dimensional Hilbert space $H$, any orthonormal basis $\left\{\left|\phi_{i}\right\rangle\right\}_{i}$ defines a commutative abstract $\mathrm{C}^{*}$-algebra on $H$ via the Schur product. This can be expressed in Dirac 'bra-ket' notation as follows:

$$
\hat{\lambda}_{\hat{R}}:=\sum_{i}\left|\phi_{i}\right\rangle\left(\left\langle\phi_{i}\left|\otimes\left\langle\phi_{i}\right|\right) \quad \hat{O}:=\sum_{i} \mid \phi_{i}\right\rangle\right.
$$

The comultiplication and counit are given as the adjoints of these two maps, namely:

$$
\mathrm{O}^{\top}:=\sum_{i}\left(\left|\phi_{i}\right\rangle \otimes\left|\phi_{i}\right\rangle\right)\left\langle\phi_{i}\right| \quad \text { ᄋ }:=\sum_{i}\left\langle\phi_{i}\right|
$$

Example 2.8. The algebra $\mathcal{B}(H)$ is almost an abstract $\mathrm{C}^{*}$-algebra, but it fails to satisfy the specialness equation by a scaling factor. However by normalising:

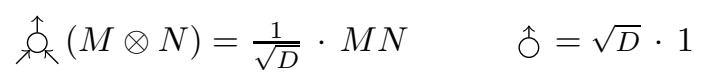

where $D$ is the dimension of $H$, we obtain an abstract $\mathrm{C}^{*}$-algebra $\mathcal{A}$ isomorphic to $\mathcal{B}(H)$. The comultiplication and counit are formed by taking the adjoint with respect to the Hilbert-Schmidt inner product on $\mathcal{B}(H)$, giving:

$$
\text { ¿ } \text { ¿ }^{\top}\left(e_{i j}\right)=\frac{1}{\sqrt{D}} \cdot \sum_{k} e_{i k} \otimes e_{k j} \quad \text { 穴 }(M)=\sqrt{D} \cdot \operatorname{tr}(M)
$$


where $e_{i j}=\left|\phi_{i}\right\rangle\left\langle\phi_{j}\right|$ for any choice of orthonormal basis of $H$. Thus, symmetry (2) captures cyclicity of the trace:

$$
\operatorname{tr}(M N)=\operatorname{tr}(N M)
$$

For any abstract $\mathrm{C}^{*}$-algebra in FHilb, we define a 'star' on elements of the algebra via:

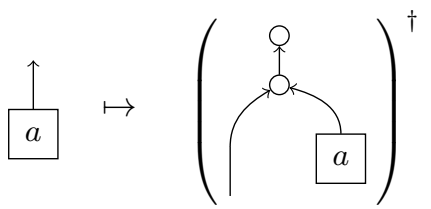

Then, the $\mathrm{C}^{*}$-algebraic norm is fixed uniquely as the spectral radius by the spectral theorem. For more details see [35].

While abstract $\mathrm{C}^{*}$-algebras correspond exactly to $\mathrm{C}^{*}$-algebras in FHilb, they correspond to a familiar, but very different kind of structure in Rel, namely groupoids.

Definition 2.9. A groupoid is a category whose morphisms are all isomorphisms.

Groupoids generalise groups, in the sense that a group is the same thing as a groupoid with a single object. Groupoids are a useful tool for capturing symmetries or other geometric information may not be fully captured by a group. For example, in homotopy theory, one can define the fundamental groupoid of a topological space without fixing a basepoint [5], which is sometimes more convenient than the fundamental group, e.g. for disconnected spaces.

Example 2.10. In the category Rel of sets and relations, special dagger Frobenius structures correspond on an object $A$ precisely to groupoids whose set of morphisms is $A$. The multiplication is the relation

$$
\text { 点 }=\{((g, f), g \circ f) \mid f, g \in A, g \circ f \text { is defined }\}
$$

and the unit is the set of identities:

$$
\hat{\wp}=\left\{\left(*, 1_{X}\right) \mid X \text { is an object in the groupoid }\right\} \subseteq I \times A
$$

The comultiplication and counit are simply the relational converses of the multiplication and unit. Finally, just like for $\mathrm{C}^{*}$-algebras in FHilb, there is a 'star' operation on elements of an abstract $\mathrm{C}^{*}$-algebra in $\mathbf{R e l}$ given by (3), which relates $g$ to its inverse $g^{-1}$. For more details see [21].

A useful calculational tool for symmetric Frobenius structures (and hence abstract $\mathrm{C}^{*}$-algebras) is the symmetric spider theorem. It tells us which of a wide variety of diagrams involving the algebraic structure are equal. Call a diagram a tree when its underlying (undirected) graph is a tree (i.e. is connected and acyclic) and planar if it contains no wire-crossings. 
Theorem 2.11 (Symmetric spider). For a symmetric dagger Frobenius structure $(A, \stackrel{\uparrow}{+}, \hat{\delta})$, suppose $f$ and $g$ can be written as planar trees consisting only of $\hat{s}$, $\hat{O}$, caps, cups, and $1_{A}$ with the same domain and codomain. Then $f=g$.

Proof. See [29].

Since any planar tree with a given input/output type is equivalent, we can collapse them into a single node without ambiguity:

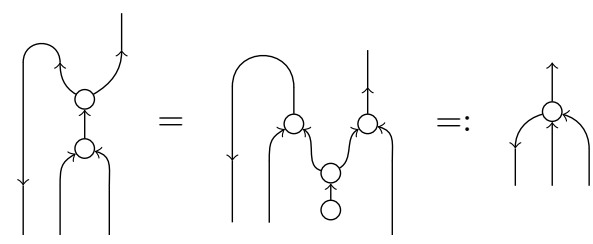

Such a node is called a spider. This generalises the spider theorem for commutative Frobenius structures, which has been widely used in the categorical quantum mechanics literature [10, 13]. However, some care needs to be taken in the symmetric case, since the restriction to planarity means that the order of inputs and outputs is relevant. So, unlike for commutative Frobenius structures:

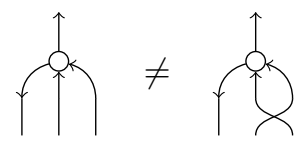

Before showing the construction of a category whose objects are all abstract $\mathrm{C}^{*}$-algebras in $\mathbf{C}$, we first focus just on the abstract analogues to $\mathrm{C}^{*}$-algebras of the form $\mathcal{B}(H)$, for a finite-dimensional Hilbert space $H$, as was done in [32]. First, we note that morphisms of the form $\rho^{\prime}: I \rightarrow H^{*} \otimes H$ are in 1-to-1 correspondence with morphisms $\rho: H \rightarrow H$. That is, there is a canonical bijection $\operatorname{hom}(H, H) \cong \operatorname{hom}\left(I, H^{*} \otimes H\right)$ given by 'bending the wire', i.e. pre-composing $\eta$ :

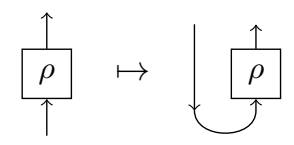

whose inverse is given by post-composing by $\epsilon$

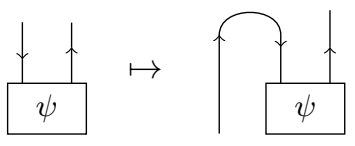

Sometimes the right-hand side of (5) is referred to as the name of the morphism $\rho$. Using it, we can represent superoperators $\operatorname{hom}(H, H) \rightarrow \operatorname{hom}(K, K)$ as morphisms of type

$$
\Phi: H^{*} \otimes H \rightarrow K^{*} \otimes K .
$$


Then, 32] showed that, when $\mathbf{C}=$ FHilb, such morphisms correspond to CPmaps precisely when they factor as

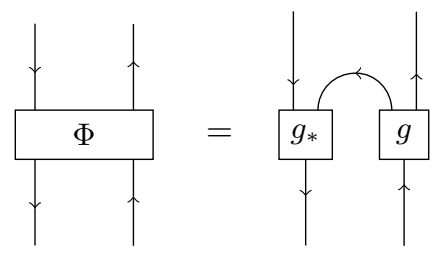

for some $g$. One way to see that this indeed gives the correct notion of CP-map in FHilb is to start with the presentation of an arbitrary CP-map in terms of the partial trace:

$$
\widetilde{\Phi}(\rho)=\operatorname{tr}_{X}\left(g^{\dagger} \rho g\right)=: \overbrace{\uparrow}^{X}
$$

and 'bend the wire' as in (5):

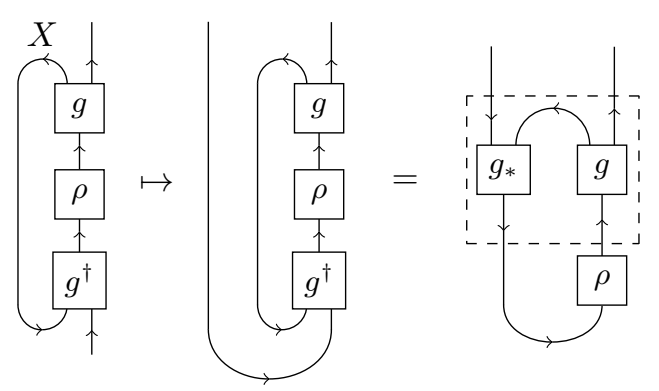

Hence, $\Phi$ depicted in (77) sends the name of $\rho$ to the name of $\widetilde{\Phi}(\rho)$ for an arbitrary CP-map $\widetilde{\Phi}$.

To pass from $\mathrm{CP}$-maps between $\mathcal{B}(H)$-type algebras to $\mathrm{CP}$-maps between arbitrary abstract $\mathrm{C}^{*}$-algebras, we can embed $\operatorname{hom}(A, B)$ into $\operatorname{hom}\left(A^{*} \otimes A, B^{*} \otimes\right.$ $B)$ as follows:

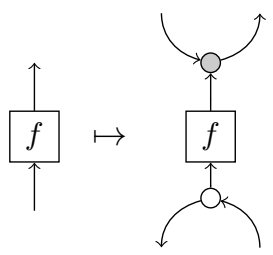

We obtain 'abstract CP-maps' by requiring that the image of this embedding satisfies (77). From this we get our main definition. 
Definition 2.12. For a compact dagger category $\mathbf{C}$, the category $\mathrm{CP}^{*}[\mathbf{C}]$ has as objects special symmetric dagger Frobenius structures ( $A$, 自), and as morphisms

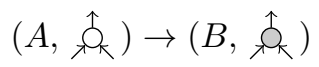

morphisms $f: A \rightarrow B$ from $\mathbf{C}$ satisfying the $C P^{*}$-condition, namely there exists a morphism $g: A \rightarrow X \otimes B$ in $\mathcal{C}$ satisfying

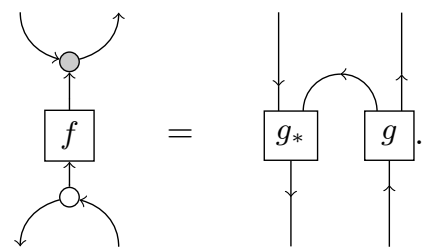

If $\mathbf{C}$ is a compact dagger category, then so is $\mathrm{CP}^{*}[\mathbf{C}]$ [12, Theorem 3.3]. Our key examples are:

- The category $\mathrm{CP}^{*}[\mathbf{F H i l b}]$, which is equivalent to the category of finitedimensional $\mathrm{C}^{*}$-algebras and completely positive linear maps [12, Proposition 3.5].

- The category $\mathrm{CP}^{*}[\mathbf{R e l}]$, which is equivalent to the category of groupoids and inverse-respecting relations [12, Proposition 5.3]. The latter are relations $R \subseteq G \times H$ between the sets of morphisms of two groupoids satisfying:

$$
\begin{aligned}
& (g, h) \in R \Longrightarrow\left(g^{-1}, h^{-1}\right) \in R, \\
& (g, h) \in R \Longrightarrow\left(1_{\operatorname{dom}(g)}, 1_{\operatorname{dom}(h)}\right) \in R .
\end{aligned}
$$

Remark 2.13. The $\mathrm{CP}^{*}$-condition comes in a variety of equivalent forms, coming from the fact that:

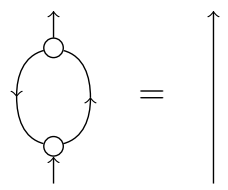

(See also [12, Lemma 2.10].) In particular, pre-composing both sides of (8) with "ơ yields

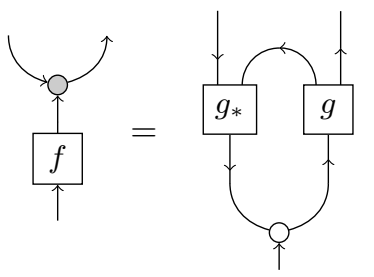

which has a familiar interpretation in FHilb. Here, $\left(A, \hat{\lambda}_{\hat{t}}\right)$ is a finite-dimensional $\mathrm{C}^{*}$-algebra whose underlying vector space $A$ happens to be a Hilbert space. 
Hence there $\left(A, \hat{\Upsilon}_{\hat{K}}\right)$ is canonically represented on $\mathcal{B}(A)$ by right-multiplication. Letting $\pi$ and $\chi$ be the representations of $\left(A, \hat{\lambda}_{\mathbb{R}}\right)$ on $\mathcal{B}(A)$ and $\left(B, \hat{\lambda}_{\mathbb{K}}\right)$ on $\mathcal{B}(B)$ respectively, condition (12) says that $f$ is a CP-map if and only if

$$
\chi(f(a))=\operatorname{tr}_{X}\left(g \pi(a) g^{\dagger}\right)
$$

for all $a \in A$, which is essentially just the Stinespring dilation of $\chi \circ f$. For finite-dimensional $\mathrm{C}^{*}$-algebras, saying that $\chi \circ f$ has a Stinesping dilation is indeed equivalent to saying that $f$ is a CP-map [7]. Combining (11) and (12) shows that $f: A \rightarrow B$ satisfies the $\mathrm{CP}^{*}$-condition if and only if

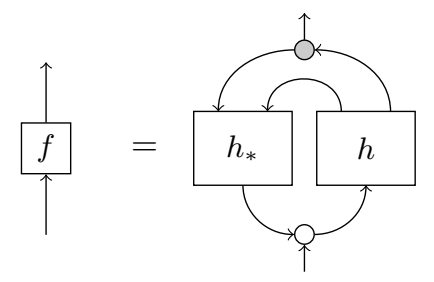

for some object $X$ and morphism $h: A \rightarrow X \otimes B$.

While we wish to remain as agnostic as possible about the base category $\mathbf{C}$, it is convenient to assume that objects $A$ in $\mathbf{C}$ are either a zero object $A \simeq 0$, or they are normalisable, i.e. there exists a positive isomorphism $\sqrt{d_{A}}: I \rightarrow I$ such that:

$$
\sqrt{d_{A}} \circ \sqrt{d_{A}}=\bigcirc A=: d_{A}
$$

We refer to $d_{A}$ as the dimension of $A$. In FHilb, this indeed gives the dimension of the Hilbert space.

We now recall some basic facts about the $\mathrm{CP}^{*}$-construction from [12]. First, there is a functor $\mathcal{B}: \mathbf{C} \rightarrow \mathrm{CP}^{*}[\mathbf{C}]$ which is analogous to the passage from a linear map $f: H \rightarrow K$ to a CP-map $\Psi_{f}: \mathcal{B}(H) \rightarrow \mathcal{B}(K)$ of the form:

$$
\Psi_{f}(\rho)=f \rho f^{\dagger}
$$

Explicitly, the functor sends an object $A$ of $\mathbf{C}$ to the special symmetric dagger Frobenius structure $A^{*} \otimes A$ with the following multiplication and unit:

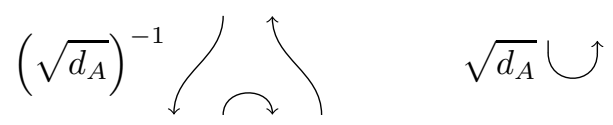

The functor sends a morphism $f$ in $\mathbf{C}$ to $f_{*} \otimes f$, which is clearly of the form (8).

Second, *-homomorphisms, i.e. morphisms in $\mathbf{C}$ satisfying:

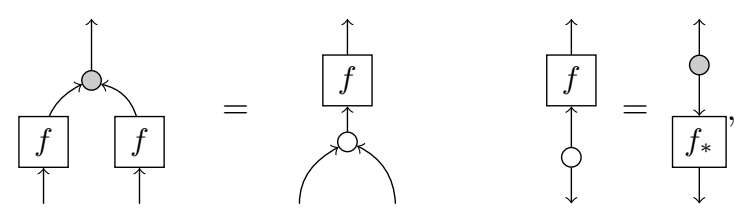


where $\hat{\underline{1}}=\hat{\hat{\Omega}_{\mathrm{k}}} \circ(1 \otimes \hat{\jmath}): A^{*} \rightarrow A$, satisfy the $\mathrm{CP}^{*}$-condition, and hence are also morphisms in $\mathrm{CP}^{*}[\mathbf{C}]$. To see this, we first give an alternative characterisation of a $*$-homomorphism.

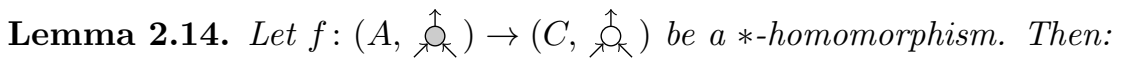
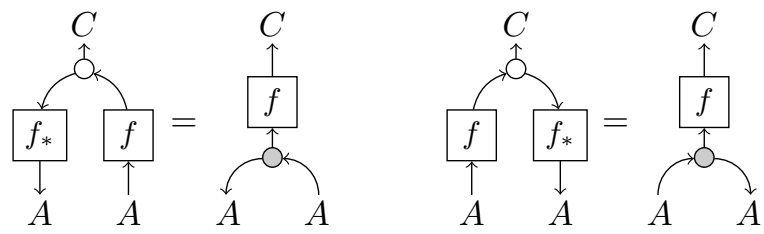

Proof. First use that $f$ preserves involution, then that it preserves multiplication:

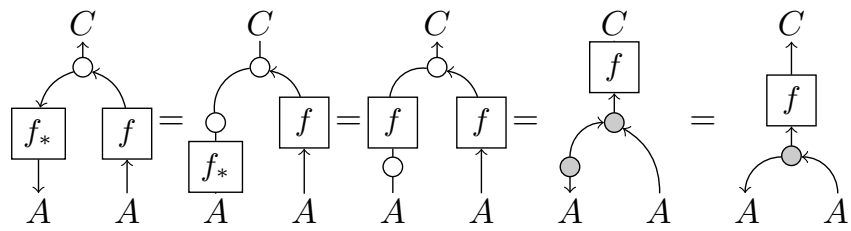

The second equations follows similarly.

The fact that $*$-homomorphisms satisfy the $\mathrm{CP}^{*}$-condition now follows immediately from Lemma 2.14 and equation (11).

The final basic concept we need before exploring the characterisation of quantum theory is the notion of causality, which tells us which processes are deterministically physically realisable.

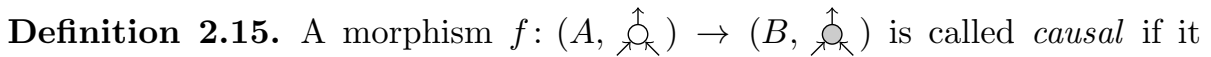
preserves co-units:

$$
\frac{9}{f}=\stackrel{\uparrow}{\uparrow}=\text { o }
$$

The causality condition says, intuitively, that if we discard the output of a process, then it doesn't matter which process happened. This condition originated in work in operational probabilistic theories [6], but was later formulated for any symmetric monoidal category whose objects come with some notion of 'discarding' [15, 9]. While it may not seem immediately obvious from its form, this requirement efficiently captures the condition that the inputs of any morphism $f$ in a string diagram can only have an effect in the causal future of $f$. That is, they can only affect the outputs of $f$, the outputs of morphisms connected to the outputs of $f$, and so on.

The abstract relationship between the causality equation and causal influence in a string diagram is explained in detail in e.g. [14, 23], but for our purposes, it suffices to consider the causality equation as a type of normalisation condition. In the case of $\mathrm{CP}$-maps $\mathcal{B}(H) \rightarrow \mathcal{B}(K)$, satisfying equation (14) 
corresponds to being trace-preserving. For more general $\mathrm{C}^{*}$-algebras, this plays a similar role to the requirement of a CP-map to be unital.

Remark 2.16. Note that our notion of causality requires a CP-map to preserve the counit, rather than unit of the $\mathrm{C}^{*}$-algebra, which is more common in the algebraic quantum information literature. This is because we work in the Schrödinger picture, where morphisms $f:\left(A, \hat{n}_{k}\right) \rightarrow\left(B, \hat{\jmath}_{k}\right)$ represent processes which take states of system $A$ to states of system $B$. Since all the categories we work with are dagger categories (and are therefore isomorphic to their opposite categories), this is equivalent to the Heisenberg picture where

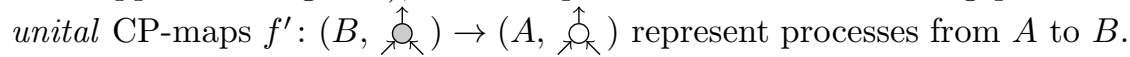

\section{Signalling}

This section focuses on the relationship between signalling and kinematic independence. To be able to capture these concepts for general $\mathrm{CP}^{*}[\mathbf{C}]$, we need to introduce the notion of subsystem.

Definition 3.1. Let $(C, \overbrace{\uparrow})$ be an abstract $\mathrm{C}^{*}$-algebra. A subsystem is another abstract $\mathrm{C}^{*}$-algebra $\left(A, \hat{\mathrm{N}}_{\mathrm{K}}\right)$ together with a morphism $f: A \rightarrow C$ satisfying $f^{\dagger} \circ f=1_{A}$ that is a unital $*$-homomorphism. We call $f$ the inclusion of the subsystem, and depict it as $\left.\right|_{A} C$.

Remark 3.2. For $f=\uparrow_{A}^{C}=1_{A} \otimes \hat{\bullet}$ we actually have that $f^{\dagger} \circ f=d_{B} \otimes 1_{A}$, rather than $1_{A}$. We can fix this by re-normalising to $\left(\sqrt{d_{B}}\right)^{-1} \otimes f$. For simplicity, we will generally suppress such normalisation factors unless they are important.

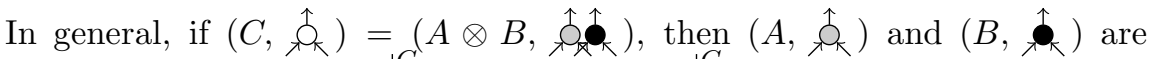
subsystems with inclusions $\nabla_{A}^{C}=1_{A} \otimes \hat{\boldsymbol{\delta}}$ and $\boldsymbol{\gamma}_{B}^{C}=\hat{\mathrm{O}} \otimes 1_{B}$. But there can also be subsystems that are not tensor factors. For $\mathbf{C}=$ FHilb, subsystems correspond precisely to $\mathrm{C}^{*}$-subalgebras. Next we look at subsystems for $\mathbf{C}=$ Rel.

Example 3.3. In Rel, a subsystem of a groupoid $\mathbf{G}$ consists of a groupoid $\mathbf{H}$ and a multi-valued function $R: H \rightarrow \mathcal{P}_{\neq \emptyset}(G)$ satisfying

$$
\begin{aligned}
& R\left(h^{-1}\right)=R(h)^{-1}, \\
& R\left(h \circ h^{\prime}\right)=R(h) \circ R\left(h^{\prime}\right), \\
& \bigcup_{x \in \mathbf{H}} R\left(1_{x}\right)=\bigcup_{y \in \mathbf{G}}\left\{1_{y}\right\}, \\
& R(h) \cap R\left(h^{\prime}\right)=\emptyset \Leftrightarrow h=h^{\prime} .
\end{aligned}
$$

We will call such subsystems wide subgroupoids. This is a slight abuse of terminology: the image of $R$ is an honest wide subgroupoid, but $R$ itself may map one morphism of $\mathbf{H}$ to many morphisms of $\mathbf{G}$. 
To see the above statement, let a groupoid $\mathbf{H}$ and relation $R \subseteq H \times G$ form a subsystem of G. As any relation, we may regard $R$ as a function $R: H \rightarrow \mathcal{P}(G)$. Isometry then says that $R(h) \neq \emptyset$, and that $R(h) \cap R\left(h^{\prime}\right)=\emptyset$ when $h \neq h^{\prime}$, that is (18). Hence $R$ is a multi-valued function. In these terms, $R$ being a unital *-homomorphism translates into (15)-(17).

There is some more structure to subsystems. If $g, g^{\prime} \in R(h)$ have the same codomain, then

$$
\begin{aligned}
g^{-1} \circ g^{\prime} \in R(h)^{-1} \circ R(h) & =R\left(h^{-1} \circ h\right) \\
& =R\left(1_{\mathrm{dom}(h)}\right) \\
& \subseteq\left\{1_{y} \mid y \in \mathbf{G}\right\},
\end{aligned}
$$

so $g=g^{\prime}$. Thus $R$ is in fact a single-valued function when restricted to subsets of $H$ of morphisms with common codomain.

The we call the dagger of a subsystem inclusion a subsystem restriction:

$$
\Delta=\left(\frac{p}{\gamma}\right)^{\dagger}
$$

Since subsystem inclusions are unital, restrictions are always causal:

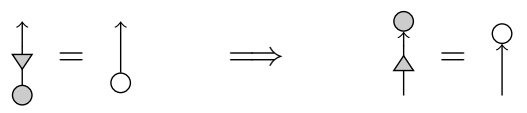

and hence physically realisable. Restrictions furthermore have a clear operational interpretation: they correspond to the process of discarding (or ignoring) everything which is not local to the subsystem $A$.

Subsystems also allow us to say when an operation on the large system is actually localised to a subsystem.

Definition 3.4. An endomorphism of $\left(C, \widehat{\lambda}_{\hat{T}}\right)$ in $\mathrm{CP}^{*}[\mathbf{C}]$ is called local to a subsystem $\left(A, \hat{\hat{N}_{\mathrm{K}}}\right)$ when it is of the form:

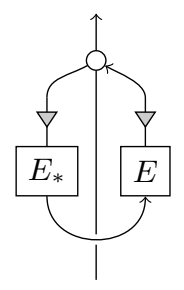

The above definition captures abstractly the $\mathrm{C}^{*}$-algebraic notion of a $\mathrm{CP}$ map only having support on a sub-algebra, i.e. a CP-map $\Phi: C \rightarrow C$ being of the form $\Phi(x)=\sum_{i} e_{i} x e_{i}^{*}$ for $e_{i}$ all elements of a sub-algebra $A$ of $C$. We will now see that when these sub-systems arise from tensor factors, this recovers the usual notion of being localised to a tensor factor, i.e. being of the form $\Phi \otimes 1$. 
Proposition 3.5. Let $\left(A, \hat{त}_{k}\right)$ and $\left(B, \hat{\gamma}_{k}\right)$ be objects in $\mathrm{CP}^{*}[\mathbf{C}]$. If a morphism $A \otimes B \rightarrow A \otimes B$ in $\mathrm{CP}^{*}[\mathbf{C}]$ is local to $A$, then it is of the form $g \otimes 1_{B}$ for

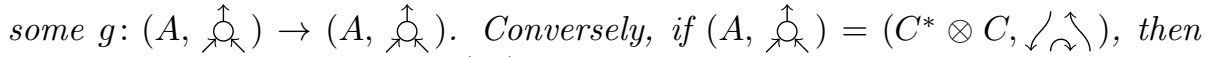
every such map has the form (19).

Proof. Unfolding Definition 3.4 shows that the morphism $A \otimes B \rightarrow A \otimes B$ has the form

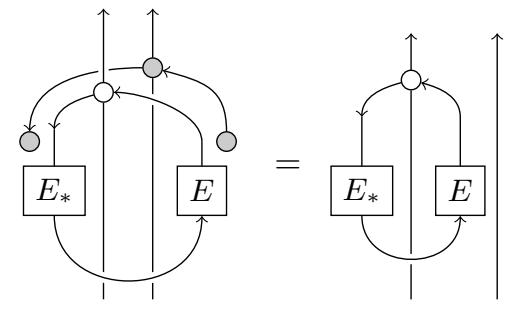

for some morphisms $E$. Thus we may take the left half of the right-hand diagram for $g$. For the converse, it suffices to show that any endomorphism of $\left(C^{*} \otimes\right.$ $C, \downarrow \curvearrowright)$ takes the desired form. That is, for any $h$, there exists $E$ such that:

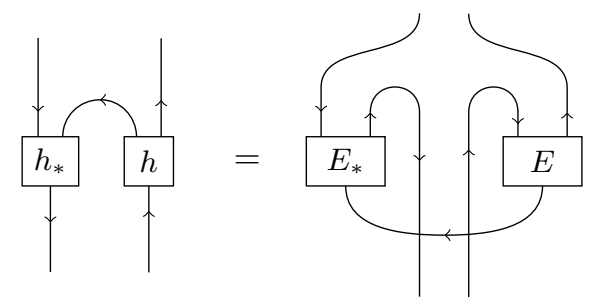

Taking

$$
\frac{\downarrow \uparrow}{t}:=\underbrace{\uparrow}
$$

this immediately follows from diagram deformation.

For the case of local maps, causality takes a simpler form.

Lemma 3.6. A map of the form (19) is causal if and only if:

$$
\text { 舟 }
$$

Proof. Causality is given by the following equation: 


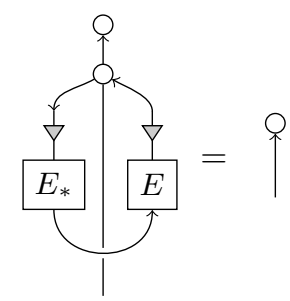

Applying a spider with two outputs to both sides yields:

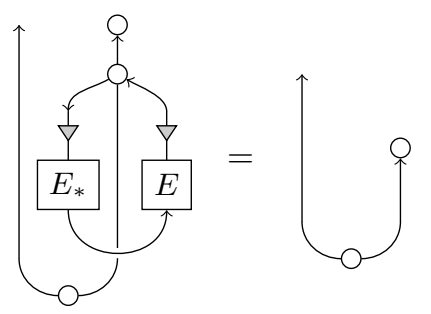

The right-hand side equals the unit, by the symmetric spider theorem. If we deform the left-hand diagram, we can also apply the symmetric spider theorem there, yielding:

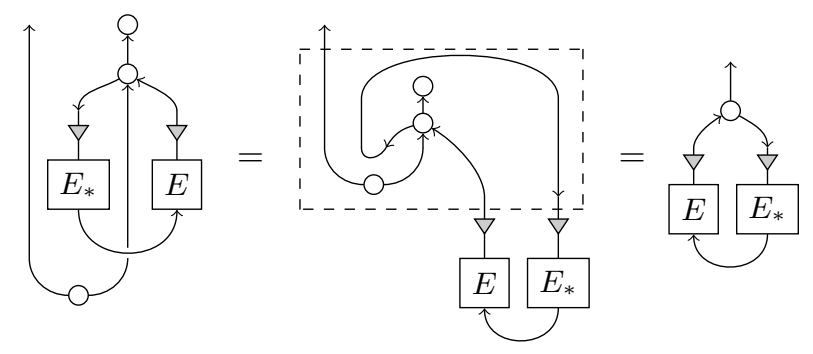

This finishes the proof.

We can now introduce the two concepts of interest. First, we consider kinematic independence. This concept formalises when systems controlled by Alice and Bob do not influence each other's kinematics, namely when the associated sub-algebras commute with respect to each other. The following definition captures this in such a way that for $\mathbf{C}=$ FHilb it coincides with the notion given in [8].

Definition 3.7. Let $\left(C, \hat{\lambda}_{\kappa}\right)$ be a dagger Frobenius structure in a compact

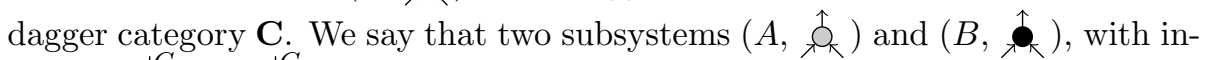
clusions $\downarrow_{A}^{C}$ and $\bigvee_{B}$, are kinematically independent when the following equation is satisfied.

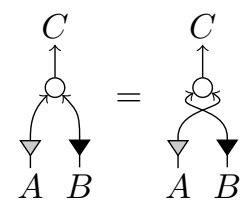


Note that, by applying the dagger to both sides, we can equivalently state kinematic indepences as:

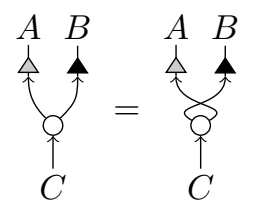

This will be important when it comes to relating this concept to nonsignalling.

Notice that if $\left(C, \hat{\lambda}_{\mathbb{R}}\right)$ decomposes into a tensor product $\left(A \otimes B, \hat{\gamma}_{\mathbf{X}}\right)$, then

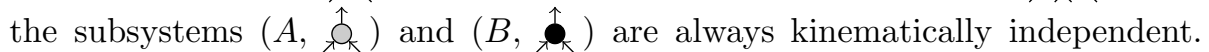
In other words, kinematic independence is a notion that essentially concerns subsystems that are not tensor factors. For example, in $\mathbf{C}=\mathbf{F H i l b}$, kinematic independence means that the $\mathrm{C}^{*}$-subalgebras $A$ and $B$ commute.

Example 3.8. Let $\mathbf{G}$ be a groupoid. Wide subgroupoids $\mathbf{A}$ and $\mathbf{B}$ are kinematically independent if and only if $S \circ T=T \circ S$ for all subsets $S \subseteq \mathbf{A}$ and $T \subseteq \mathbf{B}$ of morphisms. Equivalently, when for all subsets $S \subseteq \mathbf{A}, T \subseteq \mathbf{B}$ and elements $a \in S$ and $b \in T$, if $a \circ b$ is defined then there exist $a^{\prime} \in S, b^{\prime} \in T$ such that $a \circ b=b^{\prime} \circ a^{\prime}$, and when $b \circ a$ is defined, there exist $a^{\prime \prime} \in S, b^{\prime \prime} \in T$ such that $a^{\prime \prime} \circ b^{\prime \prime}=b \circ a$.

Proof. Plug the states $S$ and $T$ into Definition 3.7. Conversely, if $S \circ T=T \circ S$ for all states, then (201).

The next notion we consider prohibits superluminal information transfer. It says that when Alice and Bob both control a system, any data that Alice extracts from her system (through measurement) cannot instantaneously influence Bob's system. We formalise this as follows.

Definition 3.9. Let $\left(C, \hat{\lambda}_{\mathbb{K}}\right)$ be a dagger Frobenius structure in a compact

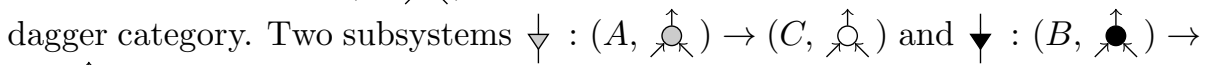
$(C, \underset{\uparrow}{\hat{\lambda}})$ are no signalling when
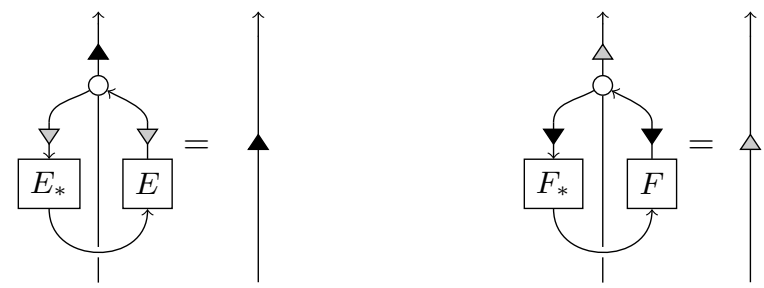

for any $E, F$ which define causal maps local to $A$ and $B$, respectively.

Again, tensor factors are automatically no signalling, making this notion essentially about subsystems that are not tensor factors: if $\left(C, \hat{p}_{\widehat{K}}\right)$ decomposes

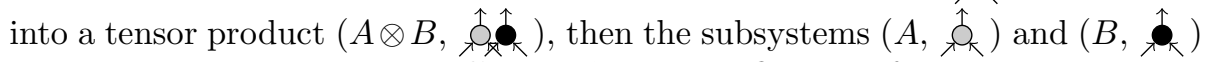
are always no signalling. For $\mathbf{C}=\mathbf{F H i l b}$, our definition of no signalling comes down to the usual one employed in [8]. 
Theorem 3.10. In $\mathrm{CP}^{*}[\mathbf{C}]$ for compact dagger $\mathbf{C}$ :

$$
\text { no signalling } \Leftrightarrow \text { kinematic independence }
$$

Proof. First, assume kinematic indepence. Then applying the symmetric spider theorem and equation (21):

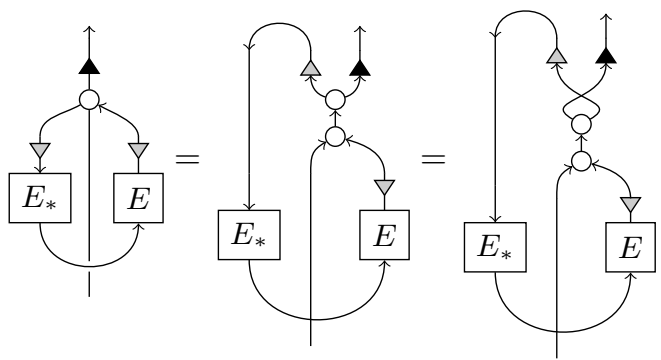

A second application of the symmetric spider theorem, as well as Lemma 3.6 yields the first no-signalling equation:

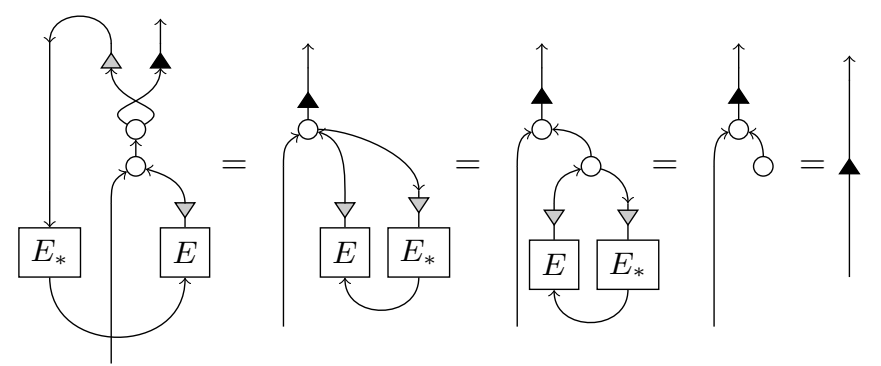

The second equation is similar.

Conversely, assume no signalling. First note that taking $E=1_{A}$ in (19) yields a causal map, by Lemma 3.6.

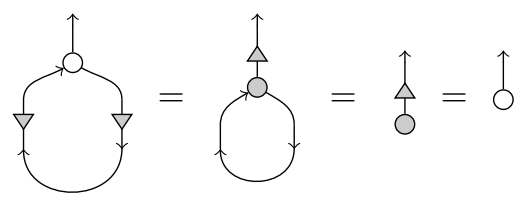

Hence the first no signalling equation from Definition 3.9 applies:

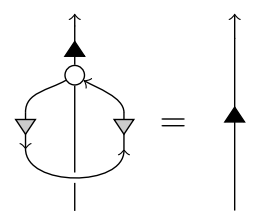

Applying this to the left-hand side of (21) introduces a loop: 


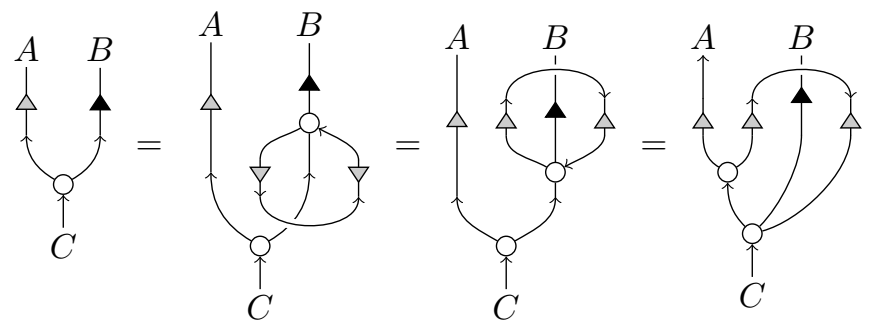

We then apply (the dagger of) the homomorphism equation:

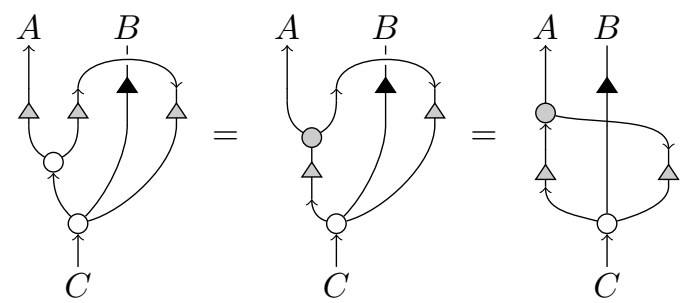

then apply the homomorphism on the other side:

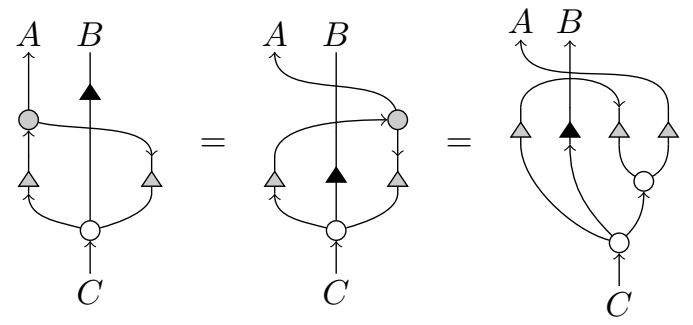

and finally remove the loop:

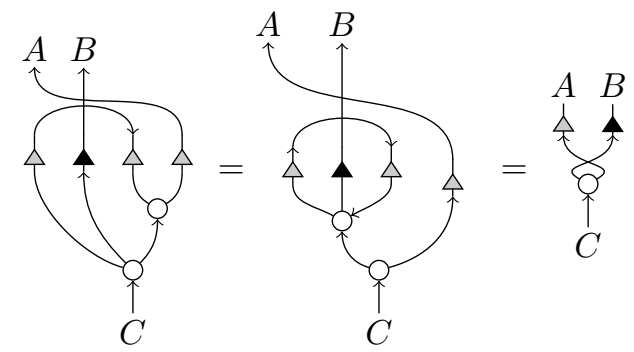

This finishes the proof.

\section{Broadcasting}

We now give a definition of broadcasting, generalising that of [8]. 


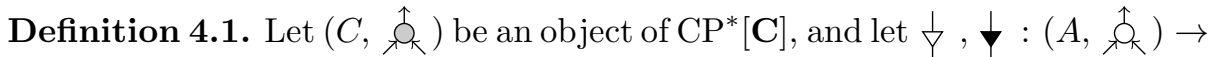

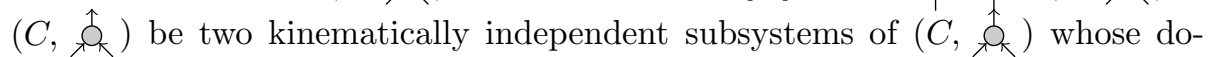
mains are a fixed algebra $\left(A, \hat{\jmath}_{-}\right)$. A broadcasting map is a morphism $B: A \rightarrow C$ in $\mathrm{CP}^{*}[\mathbf{C}]$ satisfying the following equation.

$$
\frac{a}{B}=\uparrow=\frac{\uparrow}{i}
$$

We say $A$ is broadcastable when there exists a broadcasting map for some $C$.

If $(A, \bigcirc)$ is commutative, we can simply take $C:=A$, and $\downarrow=\downarrow=$ $1_{A}$. Hence, commutatitivity trivially implies broadcastability. In the case of $\mathrm{CP}^{*}[\mathbf{F H i l b}]$, broadcastability implies commutativity, thanks to [8, Theorem 3]. However, as we will show in this section, when we pass to arbitrary $\mathrm{CP}^{*}[\mathbf{C}]$, this is no longer the case.

As in the case of concrete $\mathrm{C}^{*}$-algebras, Definition 4.1 generalises the older, more familiar notion of broadcasting presented by Barnum et al [3], which we will call $\otimes$-broadcasting.

Definition 4.2. $\mathrm{A} \otimes$-broadcasting map for an object $\left(A, \hat{\jmath}_{\mathbb{K}}\right)$ of $\mathrm{CP}^{*}[\mathbf{C}]$ is a morphism $b: A \rightarrow A \otimes A$ in $\mathrm{CP}^{*}[\mathbf{C}]$ satisfying the following equation.

$$
\frac{\stackrel{9}{\uparrow}}{\frac{i}{\uparrow}}=\uparrow=\frac{\uparrow \text { 变 }}{\uparrow}
$$

The object $(A, \underset{\uparrow}{+})$ is called $\otimes$-broadcastable when there exists a $\otimes$-broadcasting map.

Lemma 4.3. Let $\mathbf{C}$ be a compact dagger category. Commutative dagger Frobenius structures in $\mathbf{C}$ are $\otimes$-broadcastable objects in $\mathrm{CP}^{*}[\mathbf{C}]$.

Proof. Suppose that $\left(A, \widehat{\lambda}_{\boldsymbol{K}}\right)$ is commutative. We will show that "or is a broadcasting map. It clearly satisfies (23), so it suffices to show that it is a well-defined morphism in $\mathrm{CP}^{*}[\mathbf{C}]$. Using the spider theorem for commutative dagger Frobenius structures [16, Lemma 3.1], we obtain the following:

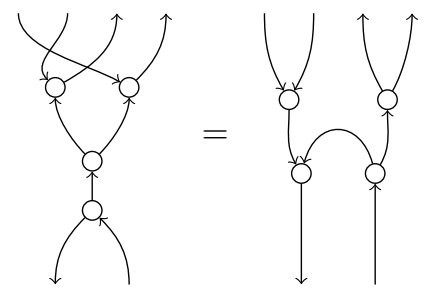


Therefore 称秀 $\mathrm{CP}^{*}[\mathbf{V}]$ and the image of "OT under the functor $\mathcal{B}$. Since these are both completely positive, so is their composition. Thus " $\mathbf{O}^{\top}$ is a well-defined morphism in $\mathrm{CP}^{*}[\mathbf{V}]$.

In particular, we can conclude that commutative $\mathrm{C}^{*}$-algebras are not only broadcastable, but also $\otimes$-broadcastable.

A groupoid is skeletal when its only morphisms are endomorphisms. Equivalently, it is a disjoint union of groups.

Lemma 4.4. $\otimes$-broadcastable objects in $\mathrm{CP}^{*}[\mathbf{R e l}]$ are precisely skeletal groupoids.

Proof. Let $\mathbf{G}$ be a skeletal (small) groupoid, and write $G$ for its set of morphisms. We will show that the morphism $b: G \rightarrow G \times G$ in Rel given by

$$
\begin{aligned}
b= & \left\{\left(f,\left(1_{\operatorname{dom}(f)}, f\right)\right) \mid f \in \operatorname{Mor}(\mathbf{G})\right\} \\
& \cup\left\{\left(f,\left(f, 1_{\operatorname{dom}(f)}\right)\right) \mid f \in \operatorname{Mor}(\mathbf{G})\right\}
\end{aligned}
$$

is a broadcasting map. First of all, $b$ is readily seen to respect identities (property (10)). Since $\mathbf{G}$ is skeletal, $1_{\operatorname{dom}(f)}=1_{\operatorname{cod}(f)}=1_{\operatorname{dom}\left(f^{-1}\right)}$, so $f$ also preserves inverses (property (9)). Hence, it is a well-defined morphism in $\mathrm{CP}^{*}[\mathbf{R e l}]$.

When interpreted in $\mathbf{R e l}$, the broadcastability equation (23) reads:

$$
\begin{aligned}
\{(f, f) \mid f \in G\} & =\left\{(f, g) \mid \exists C \cdot\left(f,\left(1_{C}, g\right)\right) \in b\right\} \\
& =\left\{(f, g) \mid \exists C \cdot\left(f,\left(g, 1_{C}\right)\right) \in b\right\} .
\end{aligned}
$$

This is satisfied by $b$ as defined above. By definition, $\left(f,\left(1_{\operatorname{dom}(f)}, f\right)\right.$ is in $b$, so the LHS of the first equation above is contained in the RHS. On the other hand, if for some $g, C$, we have $\left(f,\left(1_{C}, g\right)\right) \in b$, then $C=\operatorname{dom}(f)$ and $g=f$, so the LHS also contains the RHS. The second equation follows symmetrically.

Conversely, suppose that a small groupoid $\mathbf{G}$ is broadcastable. Then there is a morphism $b$ in $\mathbf{R e l}$ that respects inverses, and satisfies (*). Let $f \in \operatorname{Mor}(\mathbf{G})$. By $(*)$, there is an object $C$ of $\mathbf{G}$ such that $\left(f,\left(1_{C}, f\right)\right) \in b$. Next, (10) gives $\left(1_{\operatorname{dom}(f)},\left(1_{C}, 1_{\operatorname{dom}(f)}\right)\right) \in b$ and $C=\operatorname{dom}(f)$. But by (9), it is also the case that $\left(f^{-1},\left(1_{C}, f^{-1}\right)\right) \in B$. So, using $(*)$ and (10) again, we also have $\left(1_{\operatorname{cod}(f)},\left(1_{C}, 1_{\operatorname{cod}(f)}\right)\right)$ and $C=\operatorname{cod}(f)$. Hence $\operatorname{dom}(f)=\operatorname{cod}(f)$. Thus $\mathbf{G}$ is skeletal.

Lemma 4.5. Let $b$ be an $\otimes$-broadcasting map for an object $\left(A, \hat{D}_{\mathbb{R}}\right)$. Then it is also a broadcasting map, where $C:=A \otimes A, \boldsymbol{\psi}=\uparrow \hat{\mathrm{O}}$, and $\downarrow=\hat{\mathrm{O}} \uparrow$. Hence $\otimes$-broadcastability implies broadcastability.

Proof. Simply unfold the definitions.

Theorem 4.6. In $\mathrm{CP}^{*}[\mathbf{C}]$ for general $\mathbf{C}$ :

$$
\text { no broadcasting } \underset{\nLeftarrow}{\rightleftarrows} \text { noncommutativity }
$$


Proof. The implication is given by Lemmas 4.3 and 4.5. The other implication does not hold in $\mathrm{CP}^{*}[\mathbf{R e l}]$; we develop a counterexample. Let $G$ be a noncommutative group, considered as a skeletal groupoid. By Lemma 4.4, it is $\otimes$-broadcastable and hence broadcastable. However, it is noncommutative by definition.

Remark 4.7. In $\mathbf{C}=\mathbf{F H i l b}$, commutativity and broadcastability are equivalent. They also coincide with a third notion of classicality, namely that a $\mathrm{C}^{*}$ algebra is a direct sum of 1 -dimensional $\mathrm{C}^{*}$-algebras. This can be phrased for general compact dagger categories $\mathbf{C}$ with biproducts, for in that case $\mathrm{CP}^{*}[\mathbf{C}]$ inherits biproducts [22]. Hence we can consider objects in $\mathrm{CP}^{*}[\mathbf{C}]$ that arise as biproducts of the monoidal unit. One can straightforwardly show that all such objects give commutative Frobenius algebras, whereas any nontrivial abelian group in $\mathrm{CP}^{*}[\mathbf{R e l}]$ is commutative, but not a biproduct of units. Hence, we can refine Theorem 4.6 as follows:

$$
\text { biproduct of unit } \underset{\nLeftarrow}{\rightleftarrows} \text { commutative } \underset{\nLeftarrow}{\rightleftarrows} \text { broadcastable }
$$

Finally, since broadcasting coincides with commutativity when $\mathbf{C}=$ FHilb, then broadcasting and $\otimes$-broadcasting must also coincide. We leave open the question of whether this is also true for Rel.

\section{Bit commitment}

Briefly, bit commitment is the following two-party protocol. Alice claims to know something, and Bob wants to verify that Alice indeed has that knowledge, but Alice doesn't want to reveal her secret yet. Let's say the information is a single bit; Bob wants Alice to commit to either 'heads' or 'tails' now, and wants to be able to verify her committed value later. Alice could cheat by changing the value she committed to later on; if this is impossible the protocol is binding. Bob could cheat by learning the value Alice committed to before she is ready to unveil it; if this is impossible the protocol is concealing. A secure bit commitment protocol is one where neither cheat is possible. Secure bit commitment is possible under the assumption that a pair of quantum systems can only inhabit classically correlated states. Hence, impossibility of secure bit commitment implies the existence of entangled states [8]. We can model it categorically as follows.

Definition 5.1. A bit commitment protocol for a system $C$ and two subsubsystems $A, B$ of $C$ consists of two states $H, T: I \rightarrow C$ of $\mathrm{CP}^{*}[\mathbf{C}]$ which is concealing when:

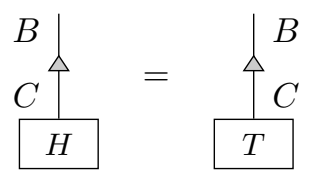


and binding when there exists no state cheat $: I \rightarrow C$ and morphisms $c_{H}, c_{T}$ localised to $A$ :
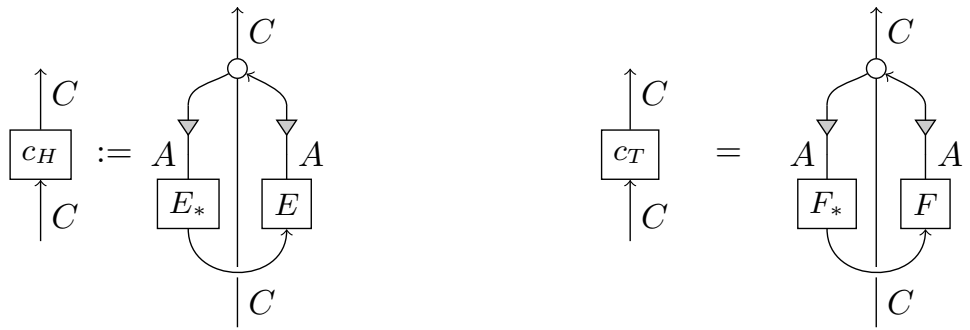

such that $c_{H} \circ$ cheat $=H$ and $c_{T} \circ$ cheat $=T$. Finally, it is secure when it is concealing, and binding.

This describes a bit commitment protocol in the following way. We assume that initially Alice has access to sub-systems $A$ and $B$, which enable her to prepare the states $H$ and $T$ of $C$. During the commitment phase of the protocol, Alice prepares either $H$ or $T$ and gives subsystem $B$ to Bob. Concealing says that at this point, Bob is unable to determine Alice's commitment. Then, for the reveal phase, Alice gives the remainder of $C$ to Bob, at which point he can ascertain whether he has state $H$ or $T$. Binding says that if Alice only has access to her own system $A$ (namely, after sending system $B$ to Bob), there is no way for Alice to change her commitment.

One thing to note here is that we have somewhat substantially limited Alice's resources if she wishes to cheat, in that she is not allowed to start with a state cheat which initially occupies a larger system, unbeknownst to Bob, and only send some part of that system in the reveal phase. This allows us to consider situations where secure bit commitment is possible, namely when Alice is unable to perform an attack using entanglement.

Example 5.2. In $\mathrm{CP}^{*}\left[\right.$ FHilb], fix the systems $B=\left(\mathbb{C}^{2},{ }_{\lambda} \hat{\gamma}_{\boldsymbol{K}}\right)$ and $Q=\left(\left(\mathbb{C}^{2}\right)^{*} \otimes\right.$ $\left.\mathbb{C}^{2}, \downarrow \uparrow\right)$ consisting of a bit and qubit. Then the states

$$
\begin{aligned}
& H=\frac{1}{2}(|0\rangle \otimes|0\rangle\langle 0|+| 1\rangle \otimes|1\rangle\langle 1|) \\
& T=\frac{1}{2}(|0\rangle \otimes|+\rangle\langle+|+| 1\rangle \otimes|-\rangle-\mid)
\end{aligned}
$$

give a (naïve) secure bit commitment protocol for $(B \otimes Q, B, Q)$. It is concealing because deleting the left system yields the maximally mixed state:

$$
(\langle+| \otimes 1) \circ H=(\langle+| \otimes 1) \circ T=\frac{1}{2}(|0\rangle\langle 0|+| 1\rangle\langle 1|)
$$

and binding because every local operation on a generic state of the form

$$
p|0\rangle \otimes \rho_{0}+q|1\rangle \otimes \rho_{1}
$$


yields a state

$$
p^{\prime}|0\rangle \otimes \rho_{0}^{\prime}+q^{\prime}|1\rangle \otimes \rho_{1}^{\prime}
$$

where $\rho_{i}^{\prime}$ is a mixture of the states $\rho_{0}, \rho_{1}$. Hence no fixed state cheat can yield both $H$ and $T$ under local operations.

We now translate the result of [8] into our language. Note that we say an object in a $\mathrm{CP}^{*}$-category is noncommutative if its associated Frobenius structure is noncommutative.

Theorem 5.3. For a system $C$ in $\mathrm{CP}^{*}[\mathbf{F H i l b}]$ with subsystems $A, B$ such that $B$ is noncommutative, the impossibility of bit commitment entails the existence of an entangled state.

Proof(sketch). It suffices to show that there exist distinct states $\rho_{1}, \rho_{2}$ of $B$ such that:

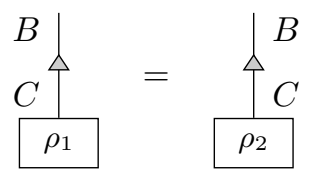

yet there exists no classically correlated state and localisable maps $c_{1}, c_{2}$ such that $c_{1} \circ \rho=\rho_{1}$ and $c_{2} \circ \rho=\rho_{2}$. This is indeed the case for any noncommutative $\mathrm{C}^{*}$-algebra. Hence, if all states $\rho$ of $C$ are classically correlated, this gives the data of a secure bit commitment protocol, and contrapositively, the impossibility of secure bit commitment necessitates the existence of an entangled state.

In [8], the authors conjectured that the converse is also true, namely that the existence of an entangled state implies the impossibility of secure bit commitment. We now show that, at least in the more general setting, this is not the case.

Proposition 5.4. The following defines a secure bit commitment protocol in Rel. Let $\mathbf{A}$ and $\mathbf{B}$ be indiscrete groupoids on 2 and 3 objects, respectively. For convenience, we name their morphisms as follows:

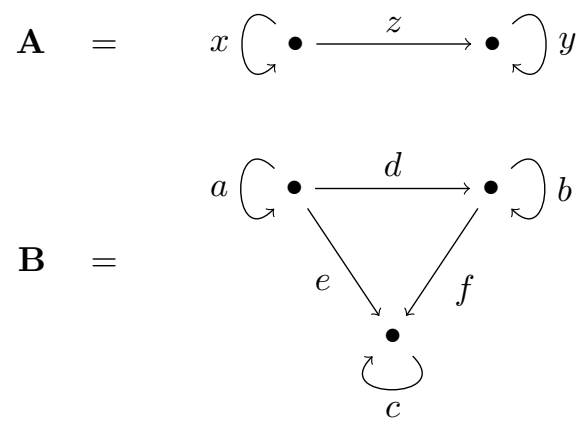


(note the inverses $d^{-1}, e^{-1}, f^{-1}$, and $z^{-1}$ are not pictured). Let $\mathbf{C}=\mathbf{A} \times \mathbf{B}$, and define the following states of $\mathbf{C}$ :

$$
\begin{aligned}
& H=(\{x\} \times \mathbf{B}) \cup\left\{(y, b),(z, d),\left(z^{-1}, d^{-1}\right)\right\}, \\
& T=(\{x\} \times \mathbf{B}) \cup\left\{(y, c),(z, e),\left(z^{-1}, e^{-1}\right)\right\} .
\end{aligned}
$$

Then, $(\mathbf{A}, \mathbf{B}, H, T)$ gives a bit commitment protocol for $\mathbf{C}$.

Proof. The key to this counterexample is that $H$ and $T$ are similar enough to be concealing, but still distinct enough that Alice cannot possibly find local maps $R$ and $S$ which produce them from a single state.

$H, T$ are closed under taking domains and inverses, so they are indeed states in $\mathrm{CP}^{*}[\mathbf{R e l}]$. To see that this is concealing, note that $(\mathrm{O} \uparrow) \circ H=($ 只 $\uparrow) \circ T=\mathbf{B}$. To see that this protocol is binding, suppose there exists a state cheat and maps $R, S: \mathbf{A} \rightarrow \mathbf{A}$ such that both $\left(R \otimes 1_{\mathbf{B}}\right) \circ$ cheat $=H$ and $\left(S \otimes 1_{\mathbf{B}}\right) \circ$ cheat $=T$. For a morphism $p \in \mathbf{A}$, let $\langle p\rangle: \mathbf{A} \rightarrow\{*\}$ be the (not necessarily inverse-respecting) relation $\{(p, *)\}$. Then, for each of the following values for $\pi: \mathbf{A} \rightarrow\{*\}$ :

$$
\langle y\rangle \circ R,\langle z\rangle \circ R,\left\langle z^{-1}\right\rangle \circ R,\langle y\rangle \circ S,\langle z\rangle \circ S,\left\langle z^{-1}\right\rangle \circ S,
$$

the composition $\left(\pi \otimes 1_{\mathbf{B}}\right) \circ$ cheat yields a distinct singleton subset of $\mathbf{B}$ (namely: $\{b\},\{d\},\left\{d^{-1}\right\},\{c\},\{e\}$, and $\left\{e^{-1}\right\}$, respectively). Hence each of these 6 effects, regarded as subsets of $\mathbf{A}$, must contain at least one element which is not in the other 5. Since $\mathbf{A}$ has only 4 morphisms, this yields a contradiction.

Definition 5.5. Let $\mathbf{C}$ be a compact dagger category, and $C$ an object in $\mathrm{CP}^{*}[\mathbf{C}]$. A state $\rho: I \rightarrow C$ is classically correlated with respect to subsystems $A$ and $B$ of $C$ when there exist a broadcastable object $X$ in $\mathrm{CP}^{*}[\mathbf{C}]$, a state $p: I \rightarrow X$, and a morphism $f: X \rightarrow C$ satisfying $\rho=f \circ p$ and:

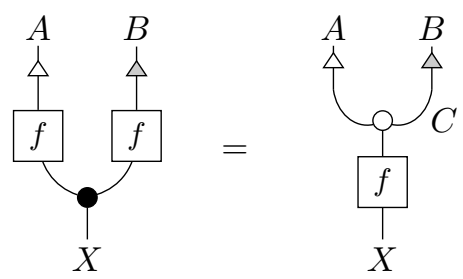

We say that $\rho$ is entangled when it is not classically correlated.

Lemma 5.6. Let $\mathbf{A}$ and $\mathbf{B}$ be wide subgroupoids of $\mathbf{C}$, regarded as subsystems in $\mathrm{CP}^{*}[\mathbf{R e l}]$, and let $R: 1 \rightarrow \mathbf{C}$ be a morphism, regarded as a subset $R \subseteq \mathbf{C}$. Suppose that $R$ classically correlates $\mathbf{A}$ and $\mathbf{B}$. If $a \circ b \in R$, then $1_{\operatorname{dom}(a)} \in R$.

Proof. Definition [5.5 unfolds as follows: there is a skeletal $G$ and maps $S: G \rightarrow$ $C$ and $P: I \rightarrow G$ with $R=P \circ S$ and such that $(g, a \circ b) \in S$ if and only if $(h, a) \in S$ and $(k, b) \in S$ and $g=h \circ k$ for some $h, k \in G$.

If $a \circ b \in R$, then there is $g \in P$ with $(g, a \circ b) \in S$. So there are $g, h \in G$ with $g \circ h \in P$ and $(g, a) \in S$ and $(b, h) \in S$. Write $e$ for identity on $\operatorname{dom}(g)=$ $\operatorname{cod}(h)$; because $G$ is skeletal also $\operatorname{dom}(h)=\operatorname{cod}(h)$ and hence $e \in P$. Then $\left(e, 1_{\mathrm{dom}(a)}\right) \in S$, and so $1_{\mathrm{dom}(a)} \in R$. 
Remark 5.7. To illustrate the slight abuse of notation in Example 3.3, let us show that in case $\mathbf{C}=\mathbf{A} \times \mathbf{B}$ the subsystems $\mathbf{A}$ and $\mathbf{B}$ are in fact multi-valued. In the setting of the previous lemma, we will show that if $(a, b) \in R$, then $\left(1_{\operatorname{dom}(a)}, 1_{\operatorname{cod}(b)}\right) \in R$.

Because $C=A \times B$, Definition 5.5 simplifies as follows: we may regard $R$ as a map $A \rightarrow B$; there is a skeletal groupoid $G$ and maps $S: G \rightarrow A \times B$ and $P: I \rightarrow G$ with:
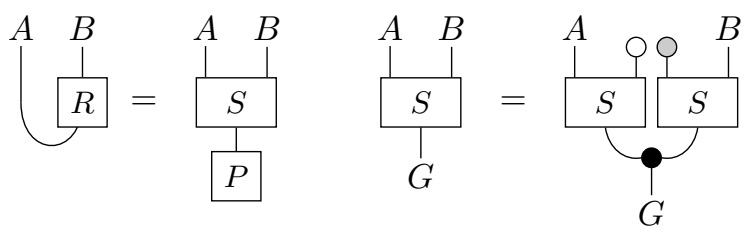

If $(a, b) \in R$ then there is $g \in P$ with $(g,(a, b)) \in S$. Hence there are $g, h \in G$ with $g \circ h \in P$ and $\left(g,\left(a, 1_{y}\right)\right) \in S$ for some object $y$ of $B$ and $\left(h,\left(1_{x}, b\right)\right) \in S$ for some object $x$ of $A$. Again the identity $e=e$ o e on $\operatorname{dom}(g)=\operatorname{cod}(h)$ is in $P$, and $\left(e,\left(1_{\operatorname{dom}(a)}, 1_{y}\right)\right) \in S$ and $\left(e,\left(1_{x}, 1_{\operatorname{cod}(b)}\right)\right) \in S$. Therefore $\left(1_{\operatorname{dom}(a)}, 1_{\operatorname{cod}(b)}\right) \in R$.

Theorem 5.8. In $\mathrm{CP}^{*}[\operatorname{Rel}]$ :

no bit commitment $\nLeftarrow$ entanglement

Proof. Proposition 5.4 exhibited a secure bit commitment protocol in $\mathrm{CP}^{*}[\mathbf{R e l}]$. It suffices to show that the system $\mathbf{C}$ defined there admits an entangled state. Let

$$
E=\left\{(x, a),(y, c),(z, e),\left(z^{-1}, e^{-1}\right)\right\}
$$

be a state of $\mathbf{C}$. Then, note that $(z, e)=(y, f) \circ(z, d)$. However, $1_{\operatorname{dom}(y, f)}=$ $(y, b) \notin E$. Hence by Lemma 5.6 (and Remark 5.6), $E$ is entangled.

The point this paper emphasises is that the impossibility of secure bit commitment is not caused by the conceptual structure of quantum theory, but by the algebraic model assumed in [8].

\section{References}

[1] S. Abramsky and B. Coecke. A categorical semantics of quantum protocols. In Logic in Computer Science 19, pages 415-425. IEEE Computer Society, 2004.

[2] H. Barnum, J. Barrett, M. Leifer, and A. Wilce. A generalized nobroadcasting theorem. Physical Review Letters, 99:240501, 2007.

[3] H. Barnum, C. M. Caves, C. A. Fuchs, R. Jozsa, and B. Schumacher. Noncommuting mixed states cannot be broadcast. Phys. Rev. Lett., 76:28182821, Apr 1996. 
[4] J. Barrett. Information processing in generalized probabilistic theories. Physical Review A, 75(3):032304, 2007.

[5] R. Brown. Topology and Groupoids. www.groupoids.org, 2006.

[6] G. Chiribella, G. M. D'Ariano, and P. Perinotti. Informational derivation of quantum theory. Physical Review A, 84(1):012311, 2011.

[7] M.-D. Choi. Completely positive linear maps on complex matrices. Linear Algebra and its Applications, 10:285-290, 1975.

[8] R. Clifton, J. Bub, and H. Halvorson. Characterizing quantum theory in terms of information-theoretic constraints. Foundations of Physics, 33(11):1561-1591, 2003.

[9] B. Coecke. Terminality implies no-signalling... and much more than that. New Generation Computing, 34(1-2):69-85, 2016.

[10] B. Coecke and R. Duncan. Interacting quantum observables: categorical algebra and diagrammatics. New Journal of Physics, 13:043016, 2011. arXiv:quant-ph/09064725.

[11] B. Coecke, C. Heunen, and A. Kissinger. Computation, Logic, Games, and Quantum Foundations. The Many Facets of Samson Abramsky: Essays Dedicated to Samson Abramsky on the Occasion of His 60th Birthday, chapter Compositional Quantum Logic, pages 21-36. Springer Berlin Heidelberg, Berlin, Heidelberg, 2013.

[12] B. Coecke, C. Heunen, and A. Kissinger. Categories of quantum and classical channels. Quantum Information Processing, 2014.

[13] B. Coecke and A. Kissinger. Categorical quantum mechanics II: classicalquantum interaction. arXiv:1605.08617 [quant-ph], 2016.

[14] B. Coecke and A. Kissinger. Picturing Quantum Processes: A First Course in Quantum Theory and Diagrammatic Reasoning. Cambridge University Press, 2017.

[15] B. Coecke and R. Lal. Causal categories: relativistically interacting processes. Foundations of Physics, 43(4):458-501, 2013.

[16] B. Coecke and É. O. Paquette. POVMs and Naimark's theorem. In $Q P L$ 2006, volume 210 of Electronic Notes in Theoretical Computer Science, pages $15-31,2006$.

[17] K. Cohn-Gordon. Commitment algorithms. Master's thesis, University of Oxford, 2012.

[18] T. Fritz. Possibilistic Physics. 2009. 
[19] M. Gachechiladze. On categorical characterizations of no-signaling theories. Master's thesis, University of Oxford, 2014.

[20] L. Hardy. Quantum theory: informational foundations and foils, chapter Reconstructing quantum theory, pages 223-248. Springer, 2016.

[21] C. Heunen, I. Contreras, and A. S. Cattaneo. Relative Frobenius algebras are groupoids. Journal of Pure and Applied Algebra, 217(1):114-124, 2013.

[22] C. Heunen, A. Kissinger, and P. Selinger. Completely positive projections and biproducts. In QPL 2014, volume 171 of Electronic Notes in Theoretical Computer Science, pages 71-83, 2014.

[23] C. Heunen and J. Vicary. Categories for Quantum Theory: An Introduction. Oxford University Press, 2019.

[24] P. A. Hoehn and C. Wever. Quantum theory from questions. arXiv preprint arXiv:1511.01130, 2015.

[25] Michael Keyl. Fundamentals of quantum information theory. Physics reports, 369(5):431-548, 2002.

[26] S. Mac Lane. Categories for the Working Mathematician. Springer, 2nd edition, 1971.

[27] L. Masanes and M. P. Müller. A derivation of quantum theory from physical requirements. New Journal of Physics, 13(6):063001, 2011.

[28] L. Masanes, M. P. Müller, R. Augusiak, and D. Pérez-García. Existence of an information unit as a postulate of quantum theory. Proceedings of the National Academy of Sciences, 110(41):16373-16377, 2013.

[29] D. Quick. !-Logic: First Order Reasoning for Families of Non-Commutative String Diagrams. PhD thesis, Oxford, 2015.

[30] M. Schlosshauer. Elegance and Enigma. Springer, 2011.

[31] Benjamin Schumacher and Michael D Westmoreland. Modal quantum theory. Foundations of Physics, 42(7):918-925, 2012.

[32] P. Selinger. Dagger compact closed categories and completely positive maps. Electronic Notes in Theoretical Computer Science, 170:139-163, 2007.

[33] P. Selinger. A survey of graphical languages for monoidal categories. In B. Coecke, editor, New Structures for Physics, Lecture Notes in Physics, pages 275-337. Springer-Verlag, 2011. arXiv:0908.3347.

[34] R. W. Spekkens. Evidence for the epistemic view of quantum states: a toy theory. Physical Review A, 75(3):032110, 2007. 
[35] J. Vicary. Categorical formulation of finite-dimensional $\mathrm{C}^{*}$-algebras. Electronic Notes in Theoretical Computer Science, 270(1):129 - 145, 2011. Proceedings of the Joint 5th International Workshop on Quantum Physics and Logic and 4th Workshop on Developments in Computational Models (QPL/DCM 2008). 